\title{
Analysis of close associations of uropod-associated proteins in human T-cells using the proximity ligation assay
}

We have shown previously that the raft-associated proteins flotillin-1 and -2 are rapidly recruited to the uropods of chemoattractant-stimulated human neutrophils and T-cells and are involved in cell polarization. Other proteins such as the adhesion receptor PSGL-1, the actin-membrane linker proteins ezrin/radixin/moesin (ERM) and the signaling enzyme phosphatidylinositol-4-phosphate 5-kinase type I gamma 90 (PIPKIgamma 90) also accumulate in the T-cell uropod. Using the in situ proximity ligation assay (PLA) we now have investigated putative close associations of these proteins in human freshly isolated T-cells before and after chemokine addition. The PLA allows in situ subcellular localization of close proximity of endogenous proteins at single-molecule resolution in fixed cells. It allows detection also of weaker and transient complexes that would not be revealed with coimmunoprecipitation approaches. We previously provided evidence for heterodimer formation of tagged flotillin-1 and -2 in T-cells before and after chemokine addition using fluorescence resonance energy transfer (FRET). We now confirm these findings using PLA for the endogenous flotillins in fixed human T-cells. Moreover, in agreement with the literature, our PLA findings confirm a close association of endogenous PSGL-1 and ERM proteins both in resting and chemokine-activated human T-cells. In addition, we provide novel evidence using the PLA for close associations of endogenous activated ERM proteins with PIPKIgamma90 and of endogenous flotillins with PSGL-1 in human T-cells, before and after chemokine addition.Our findings suggest that preformed clusters of these proteins coalesce in the uropod upon cell stimulation. 
2 Tommy Baumann ${ }^{1}$, Sarah Affentranger ${ }^{2}$ and Verena Niggli

3 From the Institute of Pathology, University of Bern, 3010 Bern, Switzerland

\begin{abstract}
4 Address for correspondence: Verena Niggli, Institute of Pathology, University of Bern, 5 Murtenstrasse 31, CH-3010 Bern, Switzerland; phone +41 3163232 35; E-mail:

6 verena.niggli@pathology.unibe.ch
\end{abstract}

7 'Present address: Department of Preventive, Restorative and Pediatric Dentistry, University of 8 Bern, Freiburgstrasse 7, 3010 Bern, Switzerland

$9{ }^{2}$ Present address: Department of Chemistry and Biochemistry, University of Bern, Freiestrasse 3, 10 CH-3012 Bern, Switzerland

11 The abbreviations used are: ERM, ezrin/radixin/moesin; FRET, fluorescence resonance energy

12 transfer; HA, hemagglutinin; PFA, paraformaldehyde, PIP-2, phosphatidylinositol-4,5-

13 bisphosphate; PIPKI $\gamma 90$, phosphatidylinositol-4-phosphate 5-kinase type I $\gamma 90$; PSGL-1, P-

14 selectin glycoprotein-1; PLA, proximity ligation assay; SDF-1, stromal cell derived factor 1;

15 TCA, trichloroacetic acid.

\title{
16 Introduction
}

17 Chemotactic migration of the highly motile T-cells is indispensable for the fulfillment of their

18 physiological functions. T-cell polarization is a prerequisite for directional migration. Polarization

19 of leukocytes requires segregation and activation of specific signaling and cytoskeletal molecules

20 in the retracting rear (uropod) and motile forward moving part (front) of the cells. Localized

21 positive feedback loops and inhibitory effects of front signaling pathways on rear signaling and

22 vice versa are thought to reinforce this structural and biochemical polarization (Friedl \& 
23 Weigelin, 2008; Bagorda \& Parent, 2008). Plasma membrane microdomains ("rafts") have been

24 implicated to stabilize polarity of migrating leukocytes (Manes \& Viola, 2006).

25 The uropod is a plasma membrane protrusion located in the rear of migrating leukocytes that 26 contains specific organelles along with cytoskeletal, adhesion and signaling proteins such as 27 activated phosphorylated ezrin/radixin/moesin proteins (P-ERM), the adhesion receptor P28 selectin glycoprotein-1 (PSGL-1) and phosphatidylinositol-4-phosphate 5-kinase type I (PIPKI) $29 \gamma 90$ (Lokuta et al., 2007; Sanchez-Madrid \& Serrador, 2009; Mathis et al., 2013). Flotillins, 30 membrane microdomain scaffolding proteins, are also enriched in leukocyte uropods and are involved in uropod formation (Ludwig et al., 2010; Rossy et al., 2009; Affentranger et al., 2011; Baumann et al., 2012). The uropod may be especially required for T-cell migration through constricted spaces (Soriano et al., 2011).

We currently study the mechanisms of targeting of specific proteins to the T-cell uropod. Transfection of human freshly isolated T-cells with a dominant-negative mutant of flotillin-2 impaired cell polarization and uropod capping of endogenous flotillin-1, PSGL-1 and GFPtagged PIPKI $\gamma 90$, indicating a functional role of flotillins in structuring the uropod (Affentranger et al., 2011; Mathis et al., 2013). Moreover, expression of constitutively active ezrin in freshly isolated T-cells induced capping of flotillins and PSGL-1, and that of a dominant-negative ezrin mutant impaired flotillin and PSGL-1 capping, suggesting a scaffolding role also for P-ERM

41 (Martinelli et al., 2013). Similarly overexpression of PIPKIy87, a naturally occurring isoform 42 lacking the last 26 amino acids, which does not locate to the uropod, suppresses T-cell uropod 43 formation and impairs capping of uropod proteins such as flotillins (Mathis et al., 2013).

44 We have now studied in situ protein-protein interactions in human T-cells fixed before and after chemokine addition, using the proximity ligation assay (PLA), in order to obtain insight into the molecular processes involved in T-cell uropod formation. The PLA allows in situ subcellular

47 localization of close proximity of proteins at single-molecule resolution (Söderberg et al., 2006).

48 It also allows detection of weaker and transient complexes that would not be revealed with co49 immunoprecipitation approaches. In contrast to fluorescence resonance energy transfer (FRET), 50 which involves expression of tagged proteins, PLA allows analysis of complexes of unmodified 51 endogenous proteins. We analyzed selected interactions of T-cell uropod-located proteins for 52 which high quality antibodies working well in immunofluorescence are available. We focused on 
53 flotillins, PSGL-1, activated ERM proteins and PIPKI $\gamma 90$. We provide novel data indicating close

54 proximity of P-ERM and PIPKI $\gamma 90$ and of flotillins and PSGL-1 in T-cells before and after

55 chemokine addition. As expected from previous data (Ivetic \& Ridley, 2004), PSGL-1 also

56 closely associates with P-ERM. Associations of flotillins with P-ERM or with PIPKI $\gamma 90$ appear

57 to be less extensive.

\section{Materials and Methods}

59 Materials and suppliers

60 Stromal cell derived factor 1 (SDF-1): Peprotech, Paris, France. Bovine serum albumin (BSA):

61 Serva, Germany. Gey's solution contained $138 \mathrm{mM} \mathrm{NaCl}, 6 \mathrm{mM} \mathrm{KCl,} 100 \mu \mathrm{M}$ EGTA, $1 \mathrm{mM}$

$62 \mathrm{Na}_{2} \mathrm{HPO}_{4}, 5 \mathrm{mM} \mathrm{NaHCO}, 5.5 \mathrm{mM}$ glucose and $20 \mathrm{mM}$ Hepes (pH 7.4).

63 Antibodies

64 Monoclonal murine antibodies directed against flotillin-2 (Cat. No. E35820) and PSGL-1 (Cat.

65 No. 556053) were obtained from Transduction Laboratories/BD Pharmingen, Germany. A

66 polyclonal rabbit antibody directed against phospho ezrin (Thr567)/ radixin (Thr564)/ moesin

67 (Thr558) (Cat. No. 3141) was from Cell Signaling Technology. Polyclonal rabbit antibodies

68 directed against flotillin-2 (Cat. No. F1680) or flotillin-1(Cat. No. F1180), a monoclonal murine

69 antibody recognizing the hemaglutinin tag (HA) (clone HA-7) and a FITC-conjugated antibody

70 directed against murine IgG (Cat. No. F5387) were obtained from Sigma. A monoclonal murine

71 antibody specifically recognizing $\beta$-cytoplasmic actin was kindly provided by $\mathrm{C}$. Chaponnier

72 (Dugina et al., 2009).

\section{Construct}

74 For preparation of N-terminally HA-tagged PIPKI $\gamma 90$, a construct coding for wild-type

75 PIP5KI 6661 N-terminally tagged with EGFP in a pcDNA3.1 vector (Lokuta et al., 2007) was

76 used as a PCR template. Then the PCR product was cloned into the phCMV2 Vector (Genlantis).

77 Isolation of human T-lymphocytes

78 Resting T-lymphocytes were isolated from buffy coats of healthy donor blood using the Pan T

79 Cell Isolation Kit II (Miltenyi Biotec) and separation on LS columns (Miltenyi Biotec) as

80 described previously (Martinelli et al., 2013). The buffy coats were obtained from the Central 
81 Laboratory of the Swiss Red Cross, Bern, Switzerland. The resulting cell suspension contained

$82>95 \%$ T-lymphocytes as assessed using anti-CD3 staining. The cells were used after over night

83 incubation in RPMI with $10 \% \mathrm{FCS}$ at $37^{\circ} \mathrm{C}$ and $5 \% \mathrm{CO}_{2}$.

\section{Immunofluorescence staining}

85 T-cells were incubated as described in the figure or table legends, followed by fixation with TCA 86 or PFA and staining for the indicated proteins as described (Affentranger et al., 2011).

\section{Transient transfections of T-lymphocytes}

88 For transfections, 3-6x $10^{6}$ freshly isolated T-lymphocytes were resuspended in $100 \mu 1$ human T cell nucleofector solution (Amaxa, Köln, Germany) diluted 1:2 with PBS and $1 \mu \mathrm{g}$ of plasmid 90 DNA was added. Then, the cell suspension was transferred to a cuvette and nucleofection was carried out (Amaxa Nucleofector, program U-14). Immediately, $500 \mu 1$ of medium with $20 \%$ FCS was added and the cells were transferred to a prewarmed 12-well plate containing $2.5 \mathrm{ml}$ of medium with $20 \% \mathrm{FCS}$, followed by incubation at $37^{\circ} \mathrm{C}$ in a $\mathrm{CO}_{2}$ incubator for $4 \mathrm{~h}$. Transfected cells were subsequently washed, resuspended in Gey's solution and used for experiments.

\section{Proximity ligation assay}

The in situ PLA (kit obtained from Olink Bioscience; www.olink.com) was used to detect protein-protein interactions in fixed cells. Nontransfected or transfected cells were treated without or with SDF-1, as described in the Figure legends, followed by fixation with TCA or PFA as described (Affentranger et al., 2011), incubation with primary antibodies, incubation with the PLA probes (anti murine and anti-rabbit IgG antibodies conjugated with oligonucleotides), ligation and amplification according to the manufacturer's instructions. Imaging was performed on fixed samples with a confocal laser scanning microscope Olympus Fluoview FV1000-IX81, 60x oil immersion objective. For determination of the fraction of cells with at least one red fluorescent dot per cell 100 cells were evaluated per sample and experiment by microscopical analysis. For the evaluation of the specific number of dots per cell and the number of dots per uropod, pictures of representative optical sections of cells with positive PLA were used (the numbers of the cells analyzed per experiment and condition are indicated in the text). 
108

109

110

\section{Results}

Interaction of flotillin-1 and -2 in freshly isolated human T-cells analyzed with the proximity ligation assay.

111 Endogenous flotillin-1 and -2 showed marked colocalization in resting and chemokine-stimulated

112 freshly isolated human T-cells (Fig. 1), as shown previously (Affentranger et al., 2011; Baumann 113 et al., 2012). Resting cells were mainly spherical, with a punctate, membrane-associated location 114 of both flotillin-1 and -2. Upon stimulation of cells with the chemokine SDF-1 for 15 minutes, 115 the majority of the cells polarized, correlating with exclusive location of both flotillins at the 116 plasma membrane at the tips of the uropods (Fig. 1A). Our previous results using FRET in cells 117 transfected with tagged flotillin-1 and -2 suggest that preformed flotillin-1 and -2

118 heterooligomers coalesce in chemokine-stimulated T-cells (Baumann et al., 2012). In the present 119 study, we have investigated interactions of endogenous flotillin-1 and -2, using the PLA, as a 120 positive control. The in situ PLA is based on fixed cells incubated with primary murine and rabbit antibodies reacting for example with flotillin-1 and -2 respectively, followed by incubation of 122 samples with modified secondary antibodies reacting with murine or rabbit IgG antibodies. These 123 secondary antibodies are conjugated with oligonucleotides (PLA probe minus and PLA probe 124 plus). Annealing of the probes occurs when the target proteins are in close proximity (less than 125 30-40 $\mathrm{nm}$ distance), which then initiates the amplification. The amplicons can be detected as red 126 dots by fluorescence microscopy (Söderberg et al., 2006). We obtained a strong PLA signal in the 127 majority of the T-cells using primary murine and rabbit antibodies to flotillin-2 and -1

128 respectively. This signal was located randomly along the plasma membrane in resting cells 129 (range: 1 - 11 dots per cell; mean: $4 \pm 1$ dots per cell; 58 cells analyzed in 3 experiments), and 130 aligned linearly around the entire border of the uropod (at least 4 dots per uropod) in $65 \%(n=2$;

13186 cells analyzed) of the stimulated cells, corresponding to the location of endogenous flotillins 132 (Fig. 1B; top panels: lower magnification; lower panels; higher magnification). These data are in 133 agreement with our FRET studies indicating heterooligomerization of tagged flotillin-1 and -2 134 (Baumann et al., 2012). Very few cells with one red dot corresponding to a positive PLA reaction 135 per cell were detected when the samples were only incubated with the flotillin-1 antibody (Fig. $1361 \mathrm{C})$. 

using PLA

139 We studied in situ interactions of endogenous flotillins with the adhesion receptors PSGL-1 and 140 activated phosphorylated ERM (P-ERM) proteins, and of PSGL-1 with P-ERM in fixed human T141 cells. Immunofluorescence pictures indeed show partial or extensive colocalization of PSGL-1 142 with P-ERM (Fig. 2A) and of flotillins with PSGL-1 (Fig. 3A) and P-ERM (Fig. 4A) in resting T143 cells and in the uropod of stimulated T-cells. We now analysed whether these colocalizations 144 correlate with close interactions using PLA in human T-cells. As a positive control we studied the 145 well established direct interaction between PSGL-1 and P-ERM using primary antibodies 146 specifically recognizing PSGL-1 and P-ERM respectively which work well in 147 immunofluorescence (Fig. 2A). As expected from previous findings (Ivetic \& Ridley, 2004), we 148 obtained positive PLA signals for PSGL- 1 and P-ERM in $94 \pm 2 \%$ of resting and $87 \pm 3 \%(n=3)$ 149 of chemokine-activated cells (Fig. 2B). In resting cells the dots indicating close proximity of the 150 proteins were randomly located at the cell periphery (range: $4-20$ dots per cell; mean: $12 \pm 1$ dot 151 per cell, analysed in 60 cells derived from 3 experiments). In stimulated cells the dots lined the 152 entire border of the uropod in $55 \pm 5 \%(\mathrm{n}=3)$ of the polarized PLA-positive cells (a total of 248 153 cells analysed). The remainder of the polarized PLA-positive cells featured 1-2 dots /uropod. A 154 negative control where the samples were only incubated with the P-ERM antibody is shown in 155 Fig. 2C.

156 A positive PLA reaction was also observed for PSGL-1 and flotillin-2 in resting and chemokine157 activated T-cells, confirming and extending the data obtained in human neutrophils using co158 immunoprecipitation of flotillin-2 and PSGL-1 (Rossy et al., 2009). Here we obtained positive 159 PLA signals in $83 \pm 2 \%(n=4)$ of the resting cells and $88 \pm 2 \%(n=4)$ of the chemokine160 stimulated T-cells (Fig. 3B), with fluorescent dots located at the plasma membrane of the resting 161 cells (range: 1 - 11 dots per cell; mean: $4 \pm 1$ dots per cell analysed in 30 cells derived from 3 162 experiments), and along the entire uropod border in $67 \%(\mathrm{n}=2 ; 198$ cells analysed) of the 163 polarized, PLA-positive stimulated cells. The remainder of the polarized PLA-positive cells

164 featured 1-2 dots /uropod. Negative controls with only the anti-PSGL-1 antibody are shown in 165 Figure 3C.

166 The PLA of flotillin-2 and P-ERM was also positive in $88 \pm 1 \%$ of the resting T-cells (range: 1 - 6 167 dots per cell; mean: $3 \pm 1$ dots per cell analysed in 59 cells derived from 2 experiments), and in $16854 \pm 8 \%$ of the stimulated cells. Especially in the stimulated cells the number of dots per cell was 169 clearly lower as compared to the result obtained for PSGL-1 and flotillin, indicating weaker, 
170 possibly transient interactions (Fig. 4B). Only $15 \pm 7 \%(\mathrm{n}=3)$ of polarized, PLA-positive cells

171 featured dots lining the border of the entire uropod (a total of 261 cells analysed). The remainder

172 of the polarized PLA-positive cells featured 1-2 dots /uropod. Negative controls using only the 173 anti-P-ERM antibody are shown in Figure 4C.

174 As a negative control we also applied the PLA assay to $\beta$-actin and flotillin-2 (supplementary Fig.

175 1). $\beta$-Actin is mainly located in protrusions at the front of polarized T-cells. Only small amounts

176 of $\beta$-actin are detectable in the uropod (supplementary Fig. 1A). Indeed we observed only in $22 \pm$

$1775 \%$ of the resting cells $(n=3)$ and in $33 \pm 5 \%(n=3)$ of the stimulated cells weakly positive PLA

178 signals $(1 \pm 0$ dots/cell; analysed in 68 cells with positive PLA derived from 3 independent

179 experiments) for $\beta$-actin and flotillin-2. Both the $\%$ of cells with positive PLA and the number of

180 dots per cell are thus clearly lower for this pair of antibodies as compared to the data shown in

181 Figures 1 - 5.In stimulated cells these dots were located outside of the uropod in 67 out of 68

182 inspected cells with positive PLA, derived from 3 independent experiments(supplementary Fig.

183 1B). Considering the uropod, this is thus a control showing zero response. The few dots detected

184 outside of the uropod may be explained by an incidental close contact of occasional flotillin

185 molecules located at the plasma membrane outside of the uropod with the abundant $\beta$-actin. The

186 negative control with only the anti- $\beta$-actin antibody is shown in the supplementary Figure $1 \mathrm{C}$.

187 Interactions of PIPKI 900 with P-ERM studied using PLA

188 GFP-tagged PIPKI $\gamma 90$ accumulates in the uropod of murine T-cells (Lokuta et al., 2007; Mathis

189 et al., 2013). We expressed an N-terminally HA-tagged PIPKI $\gamma 90$ construct in human T-cells and

190 observed colocalization of this construct with P-ERM in the uropod (Fig. 5A). We studied

191 possible interactions of PIPKI 900 with P-ERM using the PLA. As we have no highly specific

192 antibodies to PIPKI $\gamma 90$ available which work well in immunofluorescence staining, we

193 transfected cells with PIPKI 900 tagged with HA. The PLA was carried out with murine anti-HA

194 antibodies and rabbit antibodies reacting with P-ERM. To visualize the transfected cells, we

195 incubated the fixed cells with a FITC-tagged anti-murine IgG antibody that detects the HA

196 antibody, together with the PLA probes. The results are shown in Fig. 5B (top panels: lower

197 magnification; bottom panels: high magnification). We observe an extensive close association of

198 PIPKI $\gamma 90$ with P-ERM at the plasma membrane in $98 \pm 1 \%$ of the transfected resting cells

199 (range: 9 - 24 dots per cell; mean: $15 \pm 1$ dots per cell derived from 23 cells in 4 experiments).

200 Similarly the PLA was positive in $89 \pm 7 \%(n=3)$ of the transfected polarized cells, mostly 
201 restricted to the uropods and lining the uropods in $75 \pm 7 \%(n=3)$ of the cells (Fig. 5B). The

202 remainder of the polarized PLA-positive cells featured 1-2 dots /uropod. Dots indicating a

203 positive PLA occurred exclusively in the transfected cells as can be appreciated from the top

204 panels, where both transfected and untransfected cells are shown in the same picture (Fig. 5B).

205 Negative controls carried out only with the anti-HA antibody are shown in Fig. 5C. A less

206 extensive interaction was detected for PIPKI $\gamma 90$ and flotillin-2 (data not shown).

\section{Discussion}

208 In polarizing T-cells, specific signaling molecules, adhesion receptors, raft-associated proteins

209 and cytoskeletal proteins segregate into the uropod, a site where protrusion formation is locally

210 suppressed, possibly by a membrane cytoskeletal scaffold (Sanchez-Madrid \& Serrador, 2009).

211 The exact mechanism of uropod scaffolding is as yet unknown. We have recently shown that

212 flotillins as well as activated phosphorylated ERM proteins cooperate in T-cell uropod formation

213 and that they mutually enhance their uropod capping (Martinelli et al., 2013). This could be

214 explained by direct or indirect interactions of these proteins. We have now used the PLA

215 approach to study close associations of uropod-located proteins. This assay is very sensitive and

216 specific and provides a high signal to noise ratio. In contrast to FRET it can be used to study

217 close proximity of endogenous unmodified proteins in situ. Positive results obtained with the

218 PLA indicate either very close proximity of proteins for example in the same microdomains or

219 direct interactions. The question arises whether the intensity of the PLA signal is proportional to

220 the extent of protein association. This has been addressed in a study analyzing ErbB2

221 homoassociation in cell lines comparing FRET and PLA (Mocanu et al., 2011). The authors used

222 flow cytometry for quantification of PLA, correlated to the extent of labeling with fluorophore-

223 conjugated antibodies. They observed a non-linear dependence of the PLA signal on the extent of

224 protein expression and association, whereas the FRET data showed a linear correlation. Steric

225 hindrance between densely packed proximity probes may prevent enzymes from taking part in

226 the amplification process. The authors recommend the PLA approach as a semiquantitative tool

227 for measuring in situ protein associations (Mocanu et al., 2011). We thus did not attempt

228 quantification of the PLA data by flow cytometry. Evaluation of the number of dots per cell is

229 made difficult by the fact that coalescence of dots occurs, especially in the uropod but also in

230 resting cells. The number of dots detected obviously will also depend on the expression levels of

231 the interaction partners. Specificity of the reaction is indicated by the loss of signal when only

232 one of the antibodies is used for PLA. Moreover we could detect no PLA signals in the uropod 
233 when we used antibodies to flotillin-2 and $\beta$-actin, the latter protein being located mainly outside

234 of the uropod (supplementary Fig. 1).

235 Using FRET of differently tagged flotillin-1 and -2 expressed in human T-cells we previously

236 showed that these proteins form heterodimers in both resting and chemokine-stimulated cells, and

237 we can now confirm these findings for the endogenous flotillins using PLA (Fig. 1B). Another

238 positive control is the positive PLA observed for P-ERM and PSGL-1 in resting and activated T-

239 cells (Fig. 2B), in agreement with the literature indicating direct interaction of these proteins

240 obtained by in vitro methods (Ivetic \& Ridley, 2004).

241 We now provide novel data using in situ PLA in fixed human T-cells on close proximity of

242 specific uropod components. We show that flotillins appear to closely associate with PSGL-1 in

243 resting and stimulated T-cells, the reaction being restricted to the border of the uropod in

244 polarized cells, confirming and extending the data obtained previously in human neutrophils

245 using co-immunoprecipitation (Rossy et al., 2009). Flotillins and P-ERM appear to interact less

246 extensively. Flotillins could thus indirectly recruit P-ERM to the uropod via interactions with

247 PSGL-1.

248 Another T-cell uropod component is the enzyme PIPKI $\gamma 90$, which synthesizes

249

phosphatidylinositol-4,5-bisphosphate (PIP-2) and is involved in regulating uropod retraction in

250 leukocytes (Lokuta et al., 2007; Mathis et al., 2013). PIP-2 is required for ERM activation, inducing a conformational change and allowing subsequent C-terminal phosphorylation (Niggli

252 \& Rossy, 2008). Uropod-localized PIPKI $\gamma 90$ could thus result in locally enhanced PIP-2

253 production and ERM activation. Here we provide novel data suggesting that PIPKI $\gamma 90$ and P-

254 ERM are in close proximity in resting and activated T-cells. Interestingly, the isoform PIPKI $\beta$ has

255 also been reported to be targeted to the uropod of polarized neutrophils and to interact in vitro via

256 a 83 amino acid C-terminal domain with EBP50 (ERM-binding phosphoprotein 50). These 83 C-

257 terminal amino acids are not homologous in PIPKI $\gamma 90$ isoforms (Lacalle et al., 2007). PIPKI $\beta$

258 and PIPKI $\gamma 90$ may thus directly or indirectly interact with ERM proteins via differently

259 structured binding sites.

260 In summary our data suggest either direct interactions of flotillins with PSGL-1, and of PIPKI $\gamma 90$

261 with activated ERM proteins in resting and chemokine-activated T-cells and/or the presence of

262 these uropod-located proteins in the same membrane microdomains. Preformed complexes of

263 these proteins present already in resting cells could thus coalesce upon cell stimulation into the

264 uropod. Whether P-ERM and PIPKIy90 or flotillins and PSGL-1 indeed interact directly will 
265 have to be verified with other techniques. If so, the binding domains involved in interactions of

266 flotillins with PSGL-1 and of PIPKI 990 with P-ERM, and the possible regulation of these

267 interactions by posttranslational modifications will have to be explored.

\section{Acknowledgments}

269 We thank Dr. Erwin Sigel for critical reading of the manuscript, Dr. Anna Huttenlocher for a

270 generous gift of the PIP5KI $\gamma 661$ construct and Dr. C. Chaponnier for a kind gift of the anti- $\beta$ -

271 actin antibody. This work was supported by the Swiss National Science Foundation (Grant No

272 3100A_129655/1, to VN). 
273

\section{References}

274 Affentranger S, Martinelli S, Hahn J, Rossy J, Niggli V. 2011. Dynamic reorganization of

275 flotillins in chemokine-stimulated human T-lymphocytes. BMC Cell Biology 12:28.

276 Bagorda A, Parent CA. 2008. Eukaryotic chemotaxis at a glance. Journal of Cell Science $277 \quad$ 121:2621-2624.

278 Baumann T, Affentranger S, Niggli V. 2012. Evidence for chemokine-mediated coalescence of 279 preformed flotillin hetero-oligomers in human T-cells. Journal of Biological Chemistry $280 \quad 287: 39664-39672$.

281 Dugina V, Zwaenepoel I, Gabbiani G, Clément S, Chaponnier C. 2009. $\beta$ - and $\gamma$-cytoplasmic 282 actins display distinct distribution and functional diversity. Journal of Cell Science 122:29802832988.

284 Friedl P, Weigelin B. 2008. Interstitial leukocyte migration and immune function. Nature 285 Immunology 9:960-969.

286 Ivetic A, Ridley AJ. 2004. Ezrin/radixin/moesin proteins and Rho-GTPase signaling in 287 leucocytes. Immunology 112:165-176.

288 Lacalle RA, Peregril RM, Albar JP, Merino E, Martinez-A C, Merida I, Manes S. 2007. Type I 289 phosphatidylinositol 4-phosphate 5-kinase controls neutrophil polarity and directional movement. 290 Journal of Cell Biology 179:1539-1553.

291 Lokuta MA, Senetar MA, Bennin DA, Nuzzi PA, Chan KT, Ott VL, Huttenlocher A. 2007. Type 292 Igamma PIP kinase is a novel uropod component that regulates rear retraction during neutrophil 293 chemotaxis. Molecular Biology of the Cell 18:5069-5080. 
294 Ludwig A, Otto GP, Riento K, Hams E, Fallon PG, Nichols BJ. 2010. Flotillin microdomains

295 interact with the cortical cytoskeleton to control uropod formation and neutrophil recruitment.

296 Journal of Cell Biology 191:771-781.

297 Manes S, Viola A. 2006. Lipid rafts in lymphocyte activation and migration. Molecular

298 Membrane Biology 23:59-69.

299 Martinelli S, Chen EJH, Clarke F, Lyck R, Affentranger S, Burkhardt JK, Niggli V. 2013.

300 Ezrin/radixin/moesin proteins and flotillins cooperate to promote uropod formation in $\mathrm{T}$ cells.

301 Frontiers in Immunology 4:84.

302 Mathis L, Wernimont S, Affentranger S, Huttenlocher A, Niggli V. 2013. Determinants of

303 phosphatidylinositol-4-phosphate 5-kinase type I $\gamma 90$ uropod location in T-lymphocytes and its 304 role in uropod formation. PeerJ 1:e131.

305 Mocanu M-M, Váradi T, Szöllosi J, Nagy P. 2011. Comparative analysis of fluorescence 306 resonance energy transfer (FRET) and proximity ligation assay (PLA). Proteomics 11:2063-2070.

307 Niggli V, Rossy J. 2008. Ezrin/radixin/moesin: versatile controllers of signalling molecules and 308 of the cortical cytoskeleton. International Journal of Biochemistry and Cell Biology 40:344-349.

309 Rossy J, Schlicht D, Engelhardt B, Niggli V. 2009. Flotillins interact with PSGL-1 in neutrophils 310 and, upon stimulation, rapidly organize into membrane domains subsequently accumulating in 311 the uropod. PLoS One 4:e5403.

312 Sánchez-Madrid F, Serrador JM. 2009. Bringing up the rear: defining the roles of the uropod.

313 Nature Reviews of Molecular Cell Biology 10:353-359.

314 Söderberg O, Gullberg M, Jarvius M, Ridderstråle K, Leuchowius KJ, Jarvius J, Wester K,

315 Hydbring P, Bahram F, Larsson LG, Landegren U. 2006. Direct observation of individual 316 complexes in situ by proximity ligation. Nature Methods 3:995-1000. 
317 Soriano SF, Hons M, Schumann K, Kumar V, Dennier TJ, Lyck R, Sixt M, Stein JV. 2011. In vivo 318 analysis of uropod function during physiological T cell trafficking. Journal of Immunology 319 187:2356-2364.

320 


\section{Figure 1}

Interaction of flotillin-1 and -2 in human T-cells studied with PLA.

$(\mathrm{A}, \mathrm{B}) \mathrm{T}$-cells were preincubated for $30 \mathrm{~min}$ at $37^{\circ} \mathrm{C}$, followed by a further incubation for 15 min without or with $40 \mathrm{ng} / \mathrm{ml} \mathrm{SDF-1,} \mathrm{fixation} \mathrm{with} \mathrm{TCA} \mathrm{and} \mathrm{staining} \mathrm{for} \mathrm{endogenous} \mathrm{flotillin-1}$ (flo1) (rabbit polyclonal antibody) and flotillin-2 (flo2) (monoclonal murine antibody), followed by (A) fluorescently labeled anti-murine and anti-rabbit IgG second antibodies (IF) or (B) PLA probes minus and plus, ligation and amplification. (C) For negative controls, T-cells were treated as described for (B) except that the anti-flotillin-2 antibody was omitted. For (B) and (C), the top panels are overviews at lower magnification whereas in the lower panels single cells are shown at higher magnification. The pictures are representative of 3 experiments. The percentage of cells with one or more red fluorescent dots per cell was determined for 100 cells per sample and experiment (mean \pm sem of 3 experiments). Note that the majority of the cells incubated with both flo1 and flo2 antibodies exhibited several dots per cell, whereas for controls only incubated with flo1 antibody, maximally 1 dot per cell occurred. Bars, $10 \mu \mathrm{m}$. 


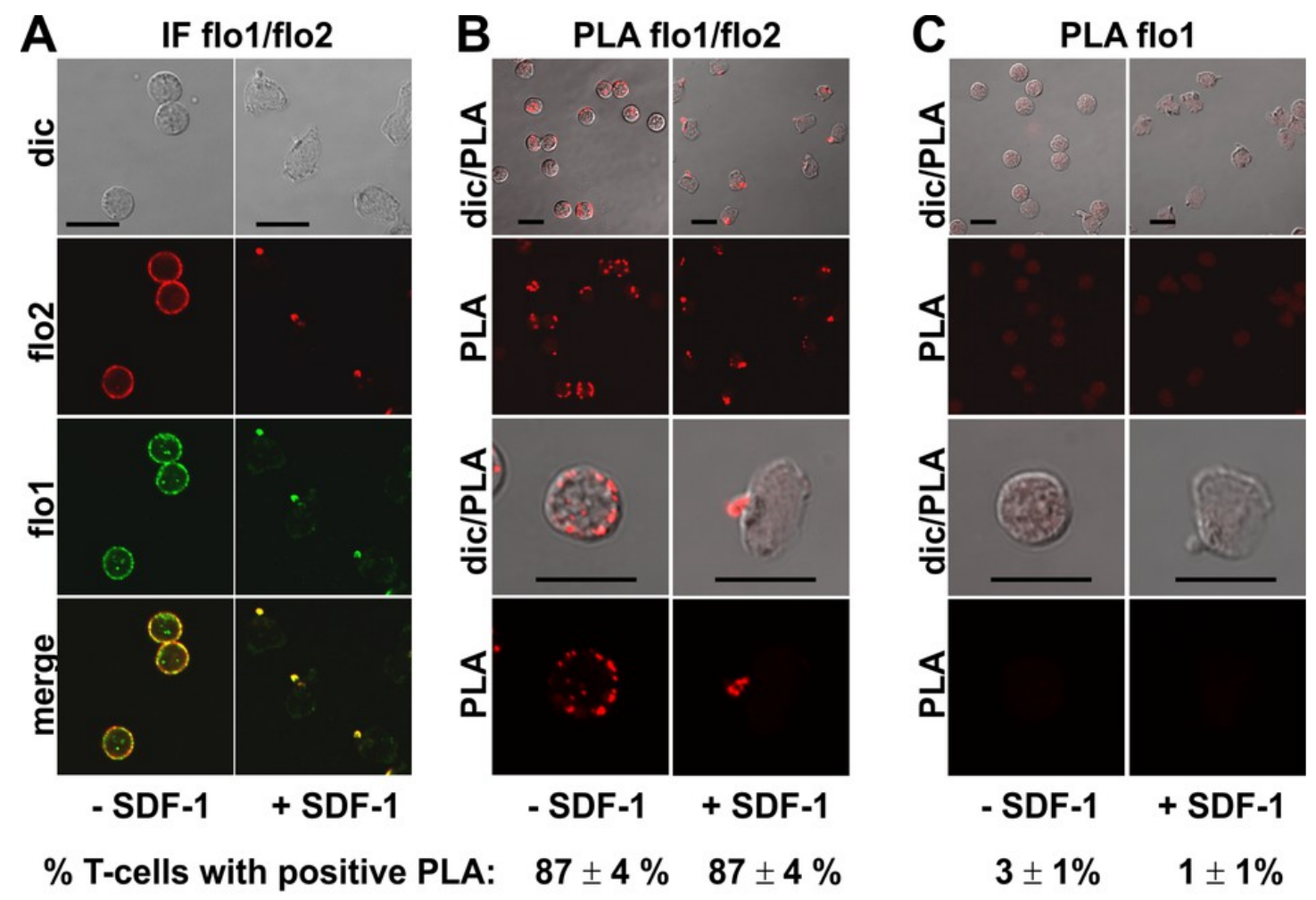




\section{Figure 2}

Interaction of PSGL-1 and P-ERM in human T-cells studied with PLA

$(A, B) \mathrm{T}$-cells were preincubated for $30 \mathrm{~min}$ at $37^{\circ} \mathrm{C}$, followed by a further incubation for 15 min without or with $40 \mathrm{ng} / \mathrm{ml}$ SDF-1, fixation with TCA and staining for endogenous PSGL-1 (monoclonal murine antibody) and P-ERM (polyclonal rabbit antibody), followed by (A) fluorescently labeled anti-murine and anti-rabbit IgG second antibodies (IF) or (B) PLA probes minus and plus, ligation and amplification. (C) For negative controls,T-cells were treated as described for (B), except that the anti-PSGL-1 antibody was omitted. For (B) and (C), the top panels are overviews at lower magnification whereas in the lower panels single cells are shown at higher magnification. The pictures are representative of 3 experiments. The percentage of cells with one or more red fluorescent dots per cell, indicating positivePLA, was determined for 100 cells per sample and experiment (mean \pm sem of 3experiments). Bars, $10 \mu \mathrm{m}$. 


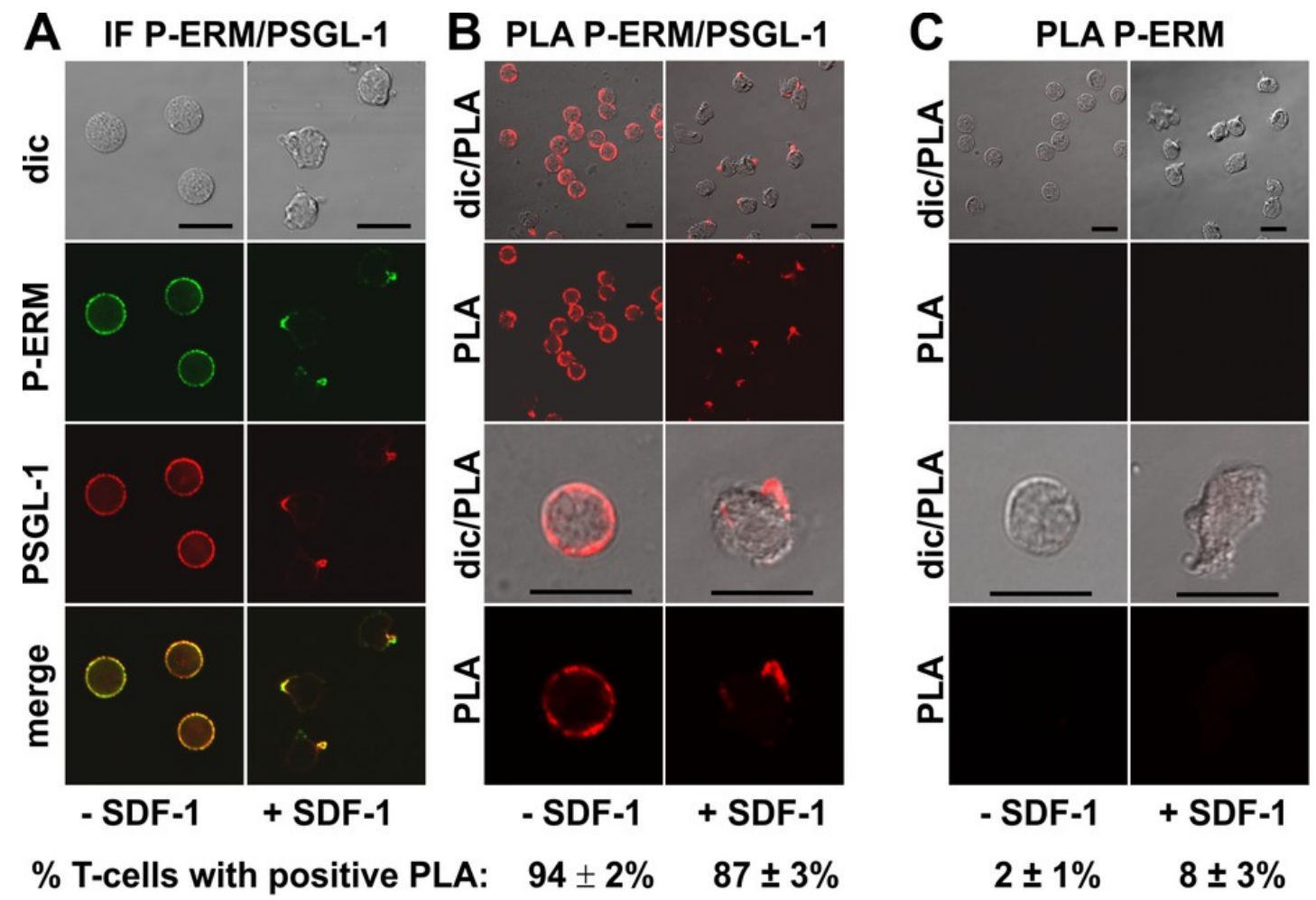




\section{Figure 3}

Interaction of PSGL-1 and flotillin-2 in human T-cells studied with PLA

$(A, B) T$-cells were preincubated for $30 \mathrm{~min}$ at $37^{\circ} \mathrm{C}$, followed by a further incubation for 15 min without or with $40 \mathrm{ng} / \mathrm{ml}$ SDF-1, fixation with TCA and staining for endogenous PSGL-1 (monoclonal murine antibody) and flotillin-2 (polyclonal rabbit antibody), followed by (A) fluorescently labeled anti-murine and anti-rabbit IgG second antibodies (IF) or (B) PLA probes minus and plus, ligation and amplification. (C) For negative controls, T-cells were treated as described for (B), except that the anti-flotillin-2 antibody was omitted. For (B) and (C),the top panels are overviews at lower magnification whereas in the lower panels single cells are shown at higher magnification. The pictures are representative of 3 experiments. The percentage of cells with one or more red fluorescent dots per cell was determined for 100 cells per sample and experiment (mean \pm sem of 3 experiments). Bars, $10 \mu \mathrm{m}$. 


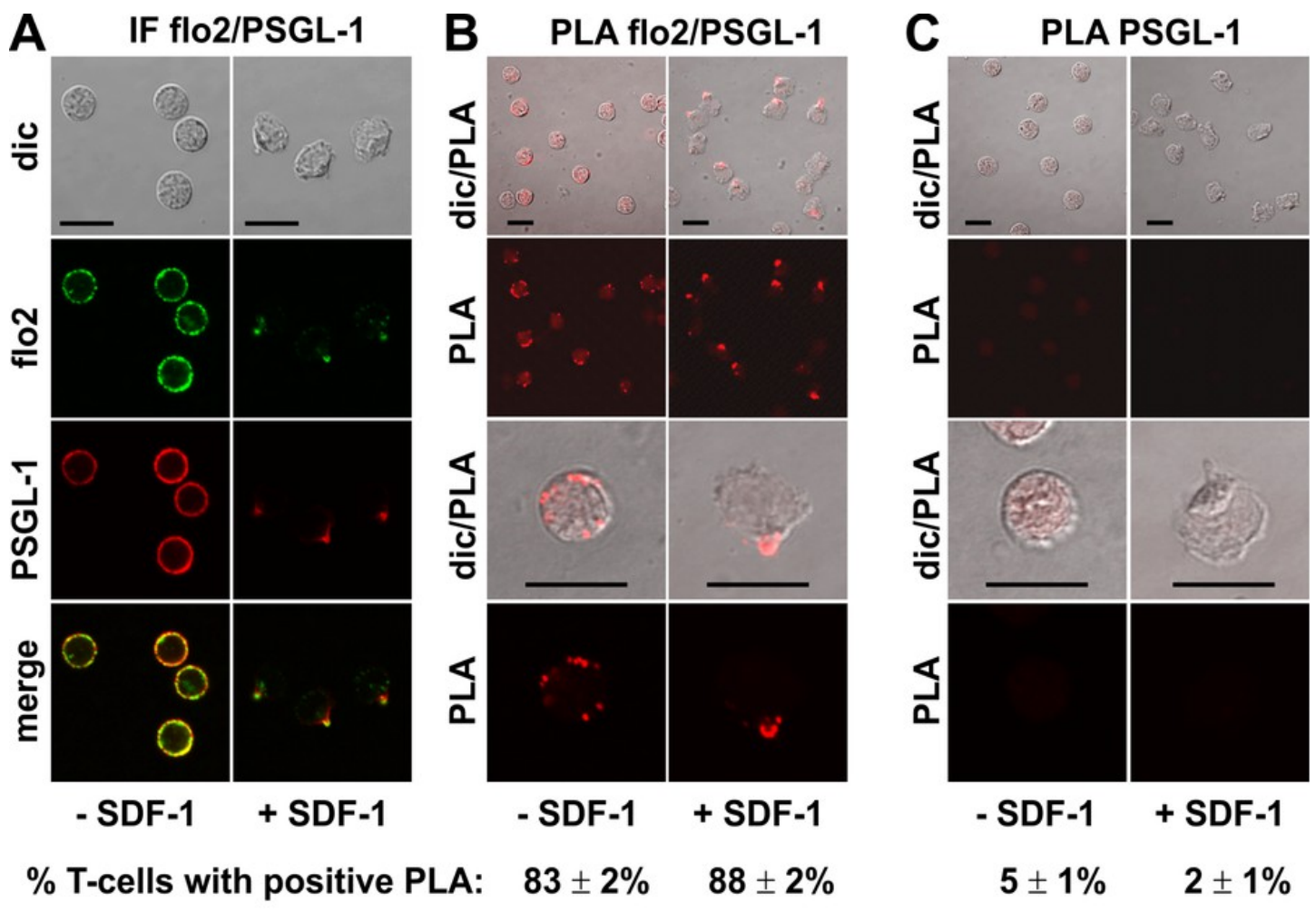




\section{Figure 4}

Interaction of P-ERM and flotillin-2 in human T-cells studied with PLA

$(A, B) T$-cells were preincubated for $30 \mathrm{~min}$ at $37^{\circ} \mathrm{C}$, followed by a further incubation for 15 min without or with $40 \mathrm{ng} / \mathrm{ml}$ SDF-1, fixation with TCA and staining for endogenous flotillin-2 (monoclonal murine antibody) and P-ERM (polyclonal rabbit antibody), followed by (A) fluorescently labeled anti-murine and anti-rabbit IgG second antibodies (IF) or (B) PLA probes minus and plus, ligation and amplification. (C) For negative controls, T-cells were treated as described for (B), except that the anti-flotillin-2 antibody was omitted. For (B) and (C), the top panels are overviews at lower magnification whereas in the lower panels single cells are shown at higher magnification. The pictures are representative of 3 experiments. The percentage of cells with one or more red fluorescent dots per cell was determined for 100 cells per sample and experiment (mean \pm sem of 3 experiments). Bars, $10 \mu \mathrm{m}$. 


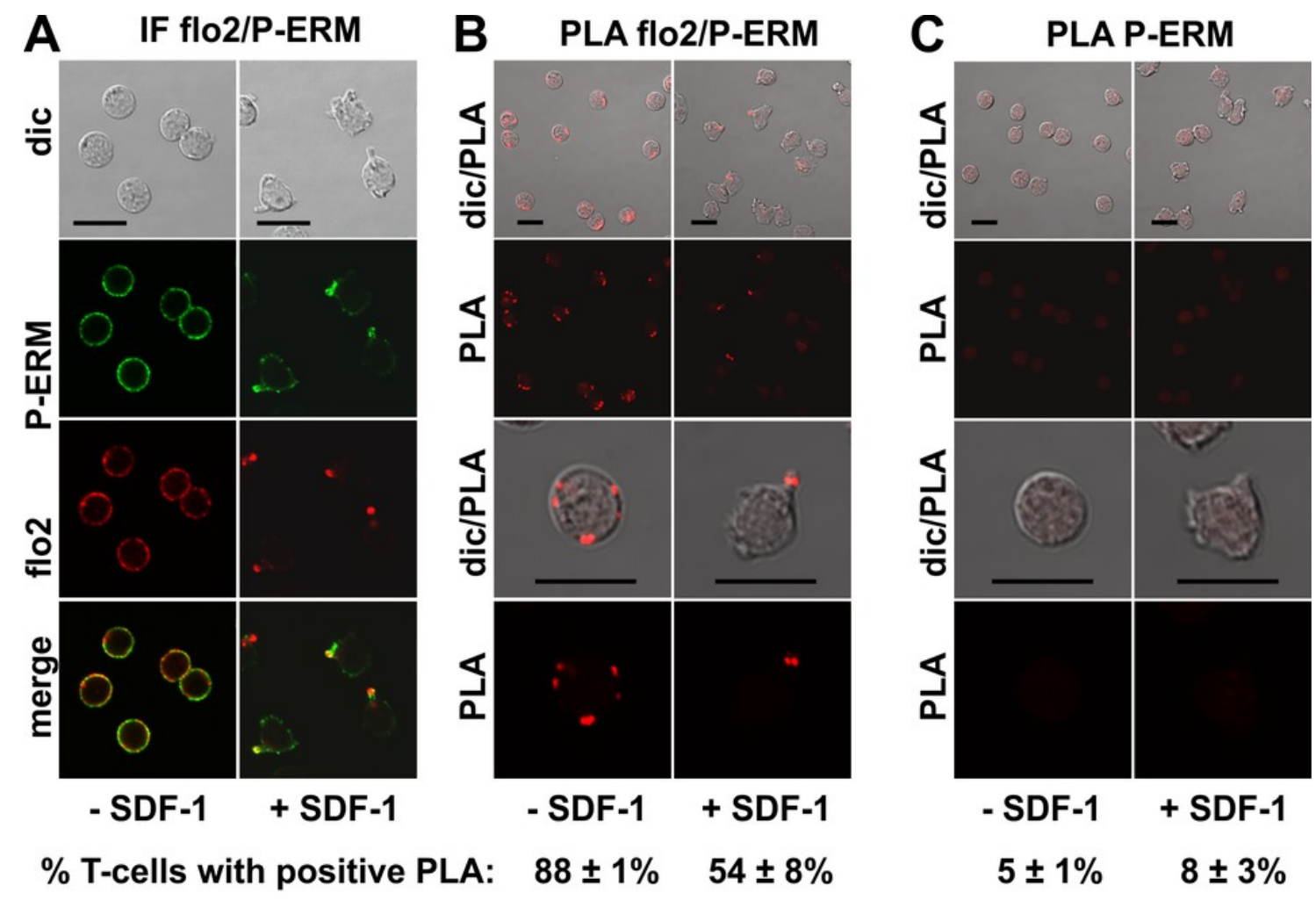




\section{Figure 5}

Interaction of PIPKIgamma90 and P-ERM in human T-cells studied with PLA.

(A-C) T-cells were transfected with HA-PIPKIgamma90. Four $h$ later the T-cells were preincubated for 30 min at $37^{\circ} \mathrm{C}$, followed by a further incubation for 15 min without or with 40 ng/ml SDF-1, fixation with TCA and staining for HA (monoclonal murine antibody) and PERM (polyclonal rabbit antibody), followed by incubation with (A) fluorescently labeled second anti-murine (red fluorescence) and anti-rabbit lgG antibodies (green fluorescence) or (B) FITC-conjugated anti-mouse IgG, in order to visualize the transfected cells (green fluorescence), and PLA probes minus and plus, ligation and amplification (red fluorescence). (C) For negative controls, transfected cells were treated as described for (B) except that the anti-P-ERM antibody was omitted. The pictures are representative of 3 experiments. For (B) and $(C)$, the top panels are overviews at lower magnification whereas in the lower panels single cells are shown at higher magnification. The percentage of transfected cells with one or more red fluorescent dots per cell was determined for 100 cells per sample and experiment (mean \pm sem for 3 experiments). Bars, $10 \mu \mathrm{m}$. 


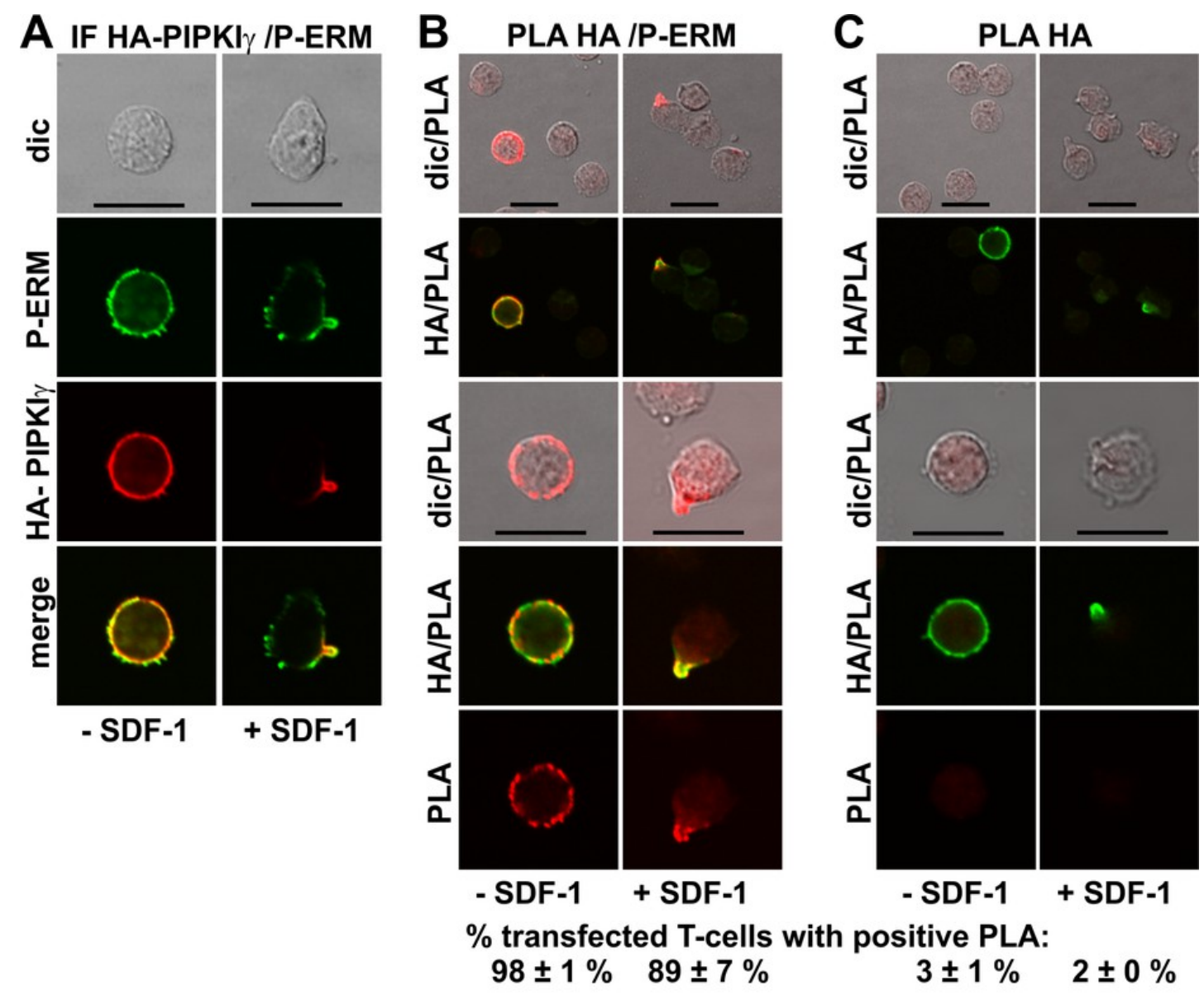

Figure 5 Peer.jeviewing PDF | (v2013:08:748:1:0:NEW 1 Oct 2013) 